\title{
Popliteal Artery Pseudoaneurysm Following Primary Total Knee Arthroplasty
}

\author{
Young-Soo Shin, MD, Yeok-Gu Hwang, MD, Abhijit Prakash Savale, MD, and Seung-Beom Han, MD \\ Department of Orthopedic Surgery, Korea University Anam Hospital, Korea University College of Medicine, Seoul, Korea
}

\begin{abstract}
An early diagnosis of popliteal artery pseudoaneurysm-a sequela of popliteal artery trauma-is difficult owing to its late presentation following total knee arthroplasty. The incidence of a popliteal artery pseudoaneurysm with a hematoma presenting only a peripheral nerve injury after total knee arthroplasty is also uncommon in the absence of common diagnostic features such as a pulsatile swelling with an audible bruit on auscultation. In the present report, we describe popliteal artery pseudoaneurysm following total knee arthroplasty.
\end{abstract}

Keywords: Popliteal artery, Pseudoaneurysm, Arthroplasty

Complications involving injury to neurological and vascular structures are infrequently reported after total knee arthroplasty (TKA). Popliteal artery pseudoaneurysm has been reported in various case studies with an incidence ranging from $0.03 \%$ to $0.17 \%^{1)}$. In addition, certain studies have indicated that the individual incidence of peroneal nerve palsy after TKA varies from $0 \%$ to $9.5 \%{ }^{2}$. Conditions associated with peroneal nerve injury after TKA include flexion contracture, valgus deformity, postoperative epidural analgesia, external leg compression, increased tourniquet duration, postoperative hematoma, and history of nerve root compression ${ }^{2}$. The current clinical diagnostic criteria for popliteal artery pseudoaneurysm are the presence of a pulsatile hematoma with an audible bruit and swelling over the popliteal region and the entire lower $\mathrm{leg}^{3)}$.

Thus, in the present report, we describe popliteal artery pseu-

Received July 19, 2013; Revised (1st) September 16, 2013;

(2nd) October 22, 2013; Accepted October 30, 2013

Correspondence to: Seung-Beom Han, MD

Department of Orthopedic Surgery, Korea University Anam Hopital,

Korea University College of Medicine, 73 Inchon-ro, Seongbuk-gu,

Seoul 136-705, Korea

Tel: +82-2-920-5692, Fax: +82-2-924-2471

E-mail: oshan@korea.ac.kr

This is an Open Access article distributed under the terms of the Creative Commons Attribution Non-Commercial License (http://creativecommons.org/licenses/by-nc/3.0/) which permits unrestricted non-commercial use, distribution, and reproduction in any medium, provided the original work is properly cited. doaneurysm in a patient with normal palpable pulses, but with swollen knee and ankle with a functional disability, intractable pain in the popliteal fossa, and weakness of ankle dorsiflexors.

\section{Case Report}

A 61-year-old woman who underwent bilateral TKA at a different institution, presented to our emergency orthopedic department on the third postoperative day with right knee and calf swelling, intolerable pain in the right lower limb, and weakness of right ankle dorsiflexors. Based on her surgery records, it was determined that the procedure involved an anterior midline skin incision followed by medial subperiosteal release along the proximal tibia via a medial parapatellar approach and implantation of a cemented knee prosthesis without tourniquet control (Fig. 1). There were no records available on the thromboembolic prophylaxis employed during the surgery, and the procedure was uneventful. She had no previous history of arterial disease or cardiovascular risk factors and trauma. However, the patient experienced weakness of right ankle dorsiflexors in the immediate postoperative period, which was diagnosed as common peroneal nerve palsy. The overall condition of the patient and the status of the right lower limb were good. Moreover, postoperative limb pulsation was present. The patient was immediately transferred to our emergency department and opioid analgesics were administered intravenously for pain relief. A thorough examination of the patient revealed a stable medical status and maintenance of vital 

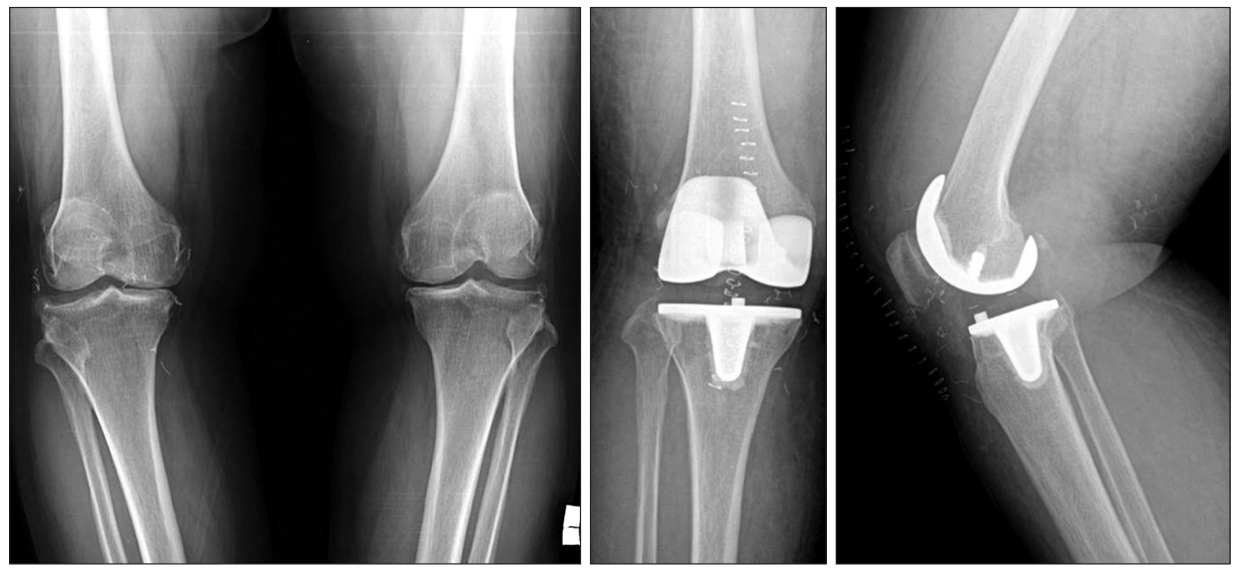

Fig. 1. Preoperative anteroposterior radiographs of both knees in a 61-year-old woman showing severe tricompartmental osteoarthritis of both knees (right>left). Immediate postoperative anteroposterior radiograph following total knee replacement of the right knee and lateral radiograph showing a well-aligned right knee with adequate cementing of components without any residual deformity.
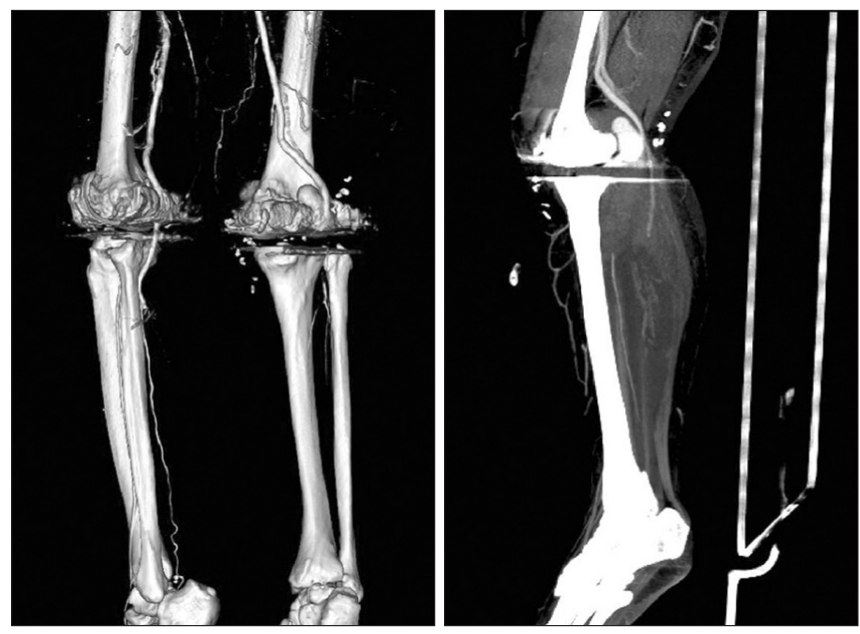

Fig. 2. Computed tomographic angiography of the right lower limb. A large pseudoaneurysm of the right popliteal artery. The popliteal artery was occluded distal to the pseudoaneurysm and was reconstituted at the tibioperoneal trunk. No significant stenosis was noted in both crural arteries with good distal runoff.

parameters. On auscultation, there were no signs of adventitious sounds such as crepitus or rhonchi. Local examination revealed swelling over the entire right lower limb with painful restriction of motion of the right knee. In addition, we also noted swelling over the popliteal region, which was non pulsatile and without any signs of auscultation bruit. The postoperative status of the wound was good with no fresh discharge. On distal neurovascular examination, the right lower limb was found to be warm, and the pulsation of both the dorsalis pedis artery and posterior tibial artery was found to be normal. Neurologically, the ankle dorsiflexors were found to have motor grade 1 , and there was a sensory deficit noted in the common peroneal nerve distribution. Based on these findings, a provisional diagnosis of common peroneal nerve palsy was made and a foot drop splint was applied
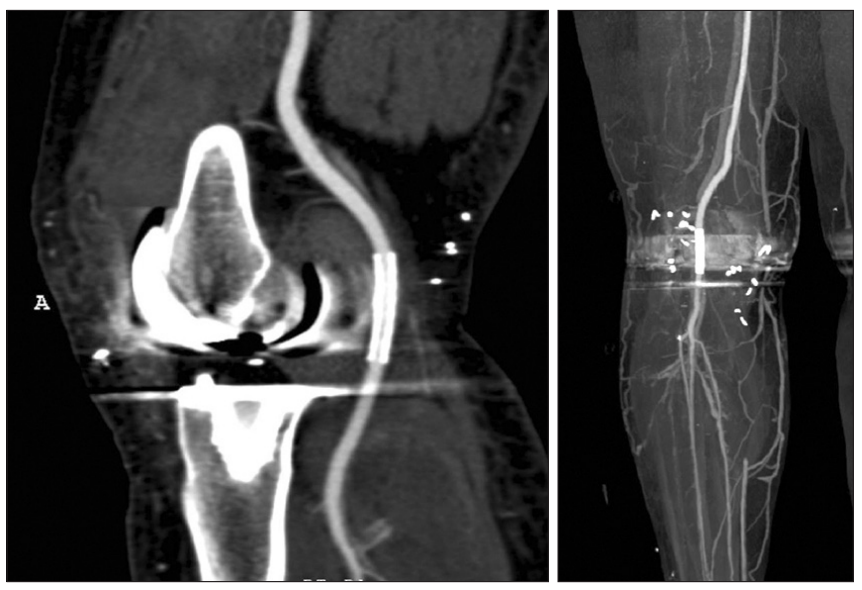

Fig. 3. A covered endovascular stent graft was placed in the right popliteal artery, and in-stent restenosis was not observed in the artery. No significant stenosis was noted in both crural arteries with good distal runoff.

immediately. The patient was then admitted to a ward for further observation. During the course of treatment, the patient reported decreased pain in the right lower limb with the use of analgesics, but the swelling of the entire right lower limb remained. A duplex examination of the right lower limb was performed as deep vein thrombosis was suspected; however, no evidence of deep vein thrombosis was noted. A computed tomographic (CT) angiography revealed a large popliteal artery pseudoaneurysm arising from the popliteal artery just above the knee with a large defect in the arterial wall, explaining the neurological symptoms (Fig. 2). The patient was immediately referred to a vascular surgeon for intervention. The lesion was treated with placement of a covered endovascular stent graft. After obtaining ante grade percutaneous access across the lesion, a covered $8 \times 30 \mathrm{~mm}$ wall graft was placed. This resulted in a satisfactory sealing of the pseudoaneurysm (Fig. 3). 
However, even 2 weeks after the onset, the peripheral neuropathies were not completely resolved and were assessed with electromyography and somatosensory evoked potential studies to document the extent of the lesion. This examination indicated evidence of denervation, thus confirming the presence of peroneal and posterior tibial nerve dysfunction; the findings were used as a baseline for subsequent studies to track recovery.

At a 6-month follow-up examination, the patient remained asymptomatic without pain or swelling; however, the patient continued to exhibit a right foot drop with normal distal pulsation.

\section{Discussion}

Popliteal artery injury most commonly occurs during the removal of osteophytes from the proximal aspect of posterior femoral condyles, during release of the posterior capsule, and during resection of the proximal tibia with an oscillating saw. The knee should be flexed during these maneuvers, thus allowing the popliteal neurovascular structures to fall posteriorly, away from the posterior capsule. The posterior midline structures should be protected, with appropriately placed retractors, when performing these maneuvers. A lever-type retractor should be placed at a sufficient distance, distally on the tibia, to facilitate protection of the neurovascular structures during translation of the proximal tibia. During resection of the posterior femoral condyles, an increased margin of safety can be gained by using a narrower saw blade and placing the retractors between the femoral condyles and collateral ligaments.

If a tourniquet is used, it should be deflated before closure. If brisk arterial bleeding is noted or if signs of distal ischemia are present, immediate consultation with a vascular surgeon is warranted. Intraoperative angiography and direct arterial repair may be necessary in such cases ${ }^{4)}$. In general, any pseudoaneurysm necessarily implies a trauma to the artery (from retractors, oscillating saw, drill, rongeur, heat from bone cement, or from repeated local trauma) $)^{5,6}$. In our case, the cause of the pseudoaneuryms also may be intraoperative trauma to the popliteal artery. However, we could not exactly reveal the apparent reasons based on her surgery records documented in another institution.

An early diagnosis of the aneurysm is very essential as it could prevent development of complications that may have serious consequences for the limb. Duplex ultrasonography is a cheap and safe diagnostic tool and is recommended, but CT angiography remains the definitive investigation to confirm the diagnosis $^{7}$. Therefore, we performed only CT angiography on the third postoperative day. However, many of these injuries are initially subclinical and remain undetected or may be misdiagnosed as postoperative hematoma or deep vein thrombosis, which are commonly occurring complications following total knee replacement ${ }^{8}$.

Geertsema et al. ${ }^{5}$, in their study of a 67 -year-old woman undergoing TKAs, found that two days postoperatively, she complained of pain at the medial part of the left ankle and plantar sensory loss of the foot explaining partial neuropathy of the peroneal and posterior tibial nerves, possibly caused by hematoma or traction. Therefore, early diagnosis is essential to avoid fatal ischemic changes of the lower extremities due to a false aneurysm and to avoid the development of persistent, irreversible, and neurological damage.

In conclusion, our study emphasized that the different presentations of popliteal artery pseudoaneurysm should be carefully considered in the absence of common diagnostic features, such as a pulsatile swelling with an audible bruit on auscultation.

\section{Conflict of Interest}

No potential conflict of interest relevant to this article was reported.

\section{References}

1. Rand JA. Vascular complications of total knee arthroplasty: report of three cases. J Arthroplasty. 1987;2:89-93.

2. Schinsky MF, Macaulay W, Parks ML, Kiernan H, Nercessian OA. Nerve injury after primary total knee arthroplasty. J Arthroplasty. 2001;16:1048-54.

3. Sloan K, Mofidi R, Nagy J, Flett MM, Chakraverty S. Endovascular treatment for traumatic popliteal artery pseudoaneurysms after knee arthroplasty. Vasc Endovascular Surg 2009;43:286-90.

4. Rama KR, Apsingi S, Poovali S, Jetti A. Timing of tourniquet release in knee arthroplasty. Meta-analysis of randomized, controlled trials. J Bone Joint Surg Am. 2007;89:699-705.

5. Geertsema D, Defoort KC, van Hellemondt GG. Popliteal pseudoaneurysm after total knee arthroplasty: a report of 3 cases. J Arthroplasty. 2012;27:1581.

6. Sandoval E, Ortega FJ, Garcia-Rayo MR, Resines C. Popliteal pseudoaneurysm after total knee arthroplasty secondary to intraoperative arterial injury with a surgical pin: review of the literature. J Arthroplasty. 2008;23:1239.

7. Boutchichi A, Ciornohac J, Daubresse F. Pseudoaneurysm after total knee arthroplasty: a rare complication with dif- 
ferent possible clinical presentations. Acta Orthop Belg. 2013;79:16-9.

8. Kobayashi S, Isobe K, Koike T, Saitoh S, Takaoka K. Acute arterial occlusion associated with total knee arthroplasty. Arch Orthop Trauma Surg. 1999;119:223-4. 